\title{
Arterial stiffness: using simple surrogate measures to make sense of a biologically complex phenomenon
}

\author{
Adam James Lewandowski, Alex Pitcher, Rajarshi Banerjee and Paul Leeson \\ Hypertension Research (2012) 35, 155-156; doi:10.1038/hr.2011.197; published online 10 November 2011
}

\section{THE IMPORTANCE OF THE PULSE}

The Ebers Papyrus from 1550 BC is the oldest description, currently identified, of how the arterial pulse can provide insights into cardiac function. ${ }^{1}$ The use of the arterial pulse to gain insight into 'stiffness' of arteries has been a relatively recent development ${ }^{2-5}$ and became possible because of the identification of two distinct waves within the arterial pulse. The first occurs in early systole as a result of direct transmission of the forward pressure from the heart along highly conductive arteries. The second is generated as the transmitted wave is reflected back from interfaces of conductive arteries with resistant arterioles. ${ }^{6}$ The general principle is that the stiffer the artery the faster the transmitted wave travels forward and the faster it is reflected, so that the gap between the forward and reflected waves at any point within the cardiovascular system shortens. A range of methods have been marketed that generate indices of arterial stiffness based on measurements of the relationship between these two waves.

TWO INDICES OF ARTERIAL STIFFNESS Clarenbach et al. ${ }^{7}$ have investigated the usefulness of two pulse wave analysis stiffness indices, one derived using a photoplethysmograph placed on a finger and, the other, from tonometry of the radial pulse. The authors clearly demonstrate that both wave reflection measures of arterial stiffness are highly repro-

$\overline{\text { Dr P Leeson and AJ Lewandowski are at the Department }}$ of Cardiovascular Medicine, Oxford Cardiovascular Clinical Research Facility, University of Oxford, John Radcliffe Hospital, Oxford OX3 9DU, UK and A Pitcher, $\mathrm{R}$ Banerjee and $\mathrm{P}$ Leeson are at the University of Oxford Centre for Clinical Magnetic Resonance Research, Department of Cardiovascular Medicine, University of Oxford, Oxford, UK

E-mail: paul.leeson@cardiov.ox.ac.uk ducible with a low failure rate in a heterogeneous population. Such characteristics make both techniques valuable for use in epidemiological studies and clinical practice. The authors also demonstrate effectively that both devices are able to differentiate individuals who have a high cumulative cardiovascular risk score, based on the EU-score, from those with a low risk score. Arterial stiffness is considered clinically important to characterize as it appears to be relevant to disease development both within the vessel wall and the heart. ${ }^{2,3,5}$ Increased velocity and augmented pulse pressure causes turbulent flow and altered shear stress within the vessel particularly at bifurcations. Furthermore, increased arterial stiffness raises the end systolic pressure required by the heart to achieve an equivalent stroke volume, accelerating cardiac hypertrophy. ${ }^{6}$ As reflected waves normally occur during the diastolic phase in healthy, compliant arteries they tend to increase coronary flow. In a stiff arterial system the earlier return of reflected waves alters perfusion of the heart. Not surprisingly, therefore, arterial stiffness has been shown in older people with risk factors to be useful as a strong predictor of future cardiovascular events, such as myocardial infarction, heart failure and stroke, as well as all-cause mortality. ${ }^{2-4}$ The paper by Clarenbach et al. would suggest both the indices they tested are able to pick out this older higher risk population.

\section{DISCREPANCIES BETWEEN TECHNIQUES}

However, the discrepancies between the two techniques, which Clarenbach et al. ${ }^{7}$ have identified, are even more interesting. Most dramatic is how inconsistent the two indices are in their relation to individual risk factors.
The digital plethysmograph index was higher in those who smoked or had a high body mass index whereas central augmentation index associated with individuals with hypertension or high total cholesterol. In view of these observations, it is not surprising that there was only a moderate association between the two indices when the cohort was considered as a whole. Furthermore, there was no association at all in subjects with low cardiovascular risk. If arterial stiffness is a continuum that affects the arterial tree uniformly it would be expected that, even at low levels of risk, measures of arterial stiffness would correlate. In the Discussion, Clarenbach et al. usefully point out that risk factors may have variable impacts on the different elements of the vasculature that contribute to arterial stiffness (Figure 1). Earlier in disease development some determinants of arterial stiffness may be more markedly affected than others by particular risk exposures. Arterial stiffness indices may then differ in which biological features of arterial stiffness they reflect.

\section{VARIABILITY IN THE PROCESS OF ARTERIAL STIFFENING}

This hypothesis is consistent with what is known about the biological process of arterial stiffening ${ }^{8}$ and clinical research that highlights regional changes in arterial stiffness 9 and selective derangement in parameters of vascular function in younger cohorts. ${ }^{9,10}$ Arterial stiffness reflects a multifactorial interplay of structural and cellular changes in the vessel wall, and is not uniform within the arterial tree. ${ }^{5}$ The structural proteins elastin and collagen are the primary mediators of arterial wall compliance and stability. ${ }^{5}$ As an artery stiffens there is an increase in abnormal collagen and reduction in normal elastin 


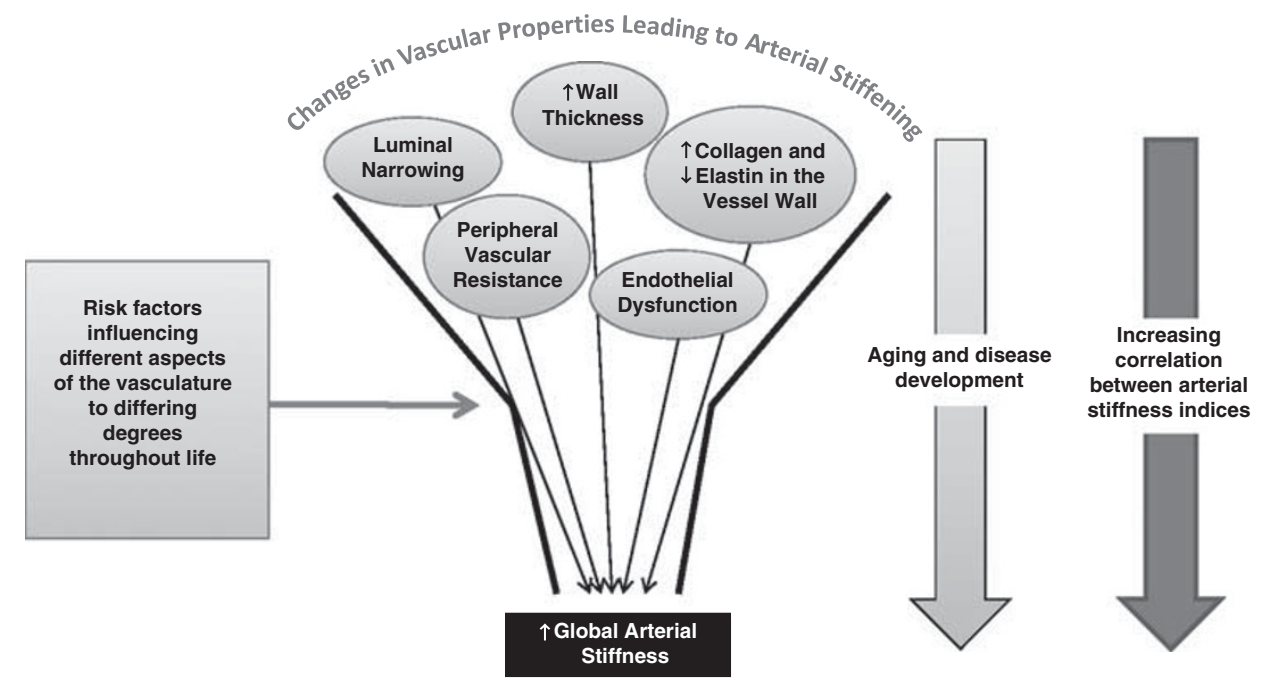

Figure 1 Convergence of arterial stiffness indices with aging and disease development. A full color version of this figure is available at the Hypertension Research journal online.

content within the vessel wall. Structural protein content varies significantly within the arterial tree, between central, elastic arteries and peripheral, muscular arteries, as well as regionally within the central arteries, and within the layers of the wall itself. A number of factors drive these changes in protein structure including inflammation, reactive oxygen species, endothelial dysfunction and increased endothelial permeability, as well as activation of certain enzymes such as matrix metalloproteinases. ${ }^{2-5}$ All these additional factors can also be measured in human studies and some used to generate their own independent vascular functional indices. Because different surrogate measures reflect different properties of the arterial wall, not all measures will detect the same specific changes induced by diseases or risk exposures in the early stages of disease development. ${ }^{3,5}$ With age and increased exposure to risk factors, such as hypertension and diabetes, a greater proportion of the arterial wall and underlying biological processes that determine arterial stiffness are affected with a resultant global increase in arterial stiffness. At this stage, all the surrogate measures of arterial stiffness would be expected to become more closely associated with each other. The two techniques Clarenbach et al. have studied, although both based on pulse wave analysis, use quite different vascular beds and different features of the pulse wave to derive their indices. Their different associa- tions with risk factors may therefore be of use to gain insight into how risk factors differentially influence disease development, the process of arterial stiffening and the pulse wave.

\section{CONCLUSIONS}

In conclusion, the use of surrogate measures of arterial stiffness is of great interest to characterize changes that may be relevant to disease development and outcome. Clarenbach et al. demonstrate that two indices, derived from digital photoplethysmography and arterial tonometry, are reproducible and likely to be easily applied in large population-based studies or clinical settings. Clarenbach et al. have also effectively demonstrated that the two different indices have quite distinct associations with individual risk factors and a lack of correlation at lower levels of risk. It is increasingly recognized that early in disease development there may be more heterogeneity and regionality in the vascular changes associated with arterial stiffening, which may explain this lack of association. More comprehensive assessment of the complex phenomenon of arterial stiffening may be required to understand this early stage of disease.

1 Nann JF. Ancient Egypt Med. British Museum Press:
2 Mitchell GF, Hwang S-J, Vasan RS, Larson MG, Pencina MJ, Hamburg NM, Vita JA, Levy D, Benjamin EJ. Arterial stiffness and cardiovascular events: The Framingham Heart Study. Circulation 2010; 121: 505-511.

3 Laurent S, Cockcroft J, Van Bortel L, Boutouyrie P, Giannattasio C, Hayoz D, Pannier B, Vlachopoulos C, Wilkinson I, Struijker-Boudier H. Expert consensus document on arterial stiffness: methodological issues and clinical applications. Eur Heart J/ 2006; 27: 2588-2605.

4 Vlachopoulos C, Aznaouridis K, Stefanadis C. Prediction of cardiovascular events and all-cause mortality with arterial stiffness: a systematic review and meta-analysis. JAm Coll Cardiol 2010; 55: 1318-1327.

5 Zieman SJ, Melenovsky V, Kass DA. Mechanisms, pathophysiology, and therapy of arterial stiffness. Arterioscler Thromb Vas Biol 2005; 25: 932-943.

6 O'Rourke MF. Pressure and flow waves in systemic arteries and the anatomical design of the arterial system. J Appl Physiol 1967; 23: 139-149.

7 Clarenbach CF, Stoewhas A-C, van Gestel AJR, Latshang TD, Lo Cascio CM, Bloch KE, Kohler M. Comparison of photoplethysmographic and arterial tonometry-derived indices of arterial stiffness. Hypertens Res 2012; 35: 228-233.

8 Vlachopoulos C, Aznaouridis K, Stefanadis C. Prediction of cardiovascular events and all-cause mortality with arterial stiffness: a systematic review and meta-analysis. J Am Coll Cardiol 2010; 55: 13181327.

9 Lazdam M, de la Horra A, Pitcher A, Mannie Z, Diesch J, Trevitt C, Kylintireas I, Contractor $H$, Singhal A, Lucas A, Neubauer S, Kharbanda R, Alp N, Kelly B, Leeson P. Elevated blood pressure in offspring born premature to hypertensive pregnancy. Hypertension 2010; 56: 159-165.

10 Lewandowski AJ, Lazdam M, Davis E, Kylintireas I, Diesch J, Francis J, Neubauer S, Singhal A, Lucas A, Kelly B, Leeson P. Short-term exposure to exogenous lipids in premature infants and long-term changes in aortic and cardiac function. Arterioscler Thromb Vasc Biol 2011; 31: 2125-2135. 